\title{
Meniscus Tear Presented as Bone Marrow Edema Manifested in Hybrid Images in Skeletal Scintigraphy: A Case Report
}

\author{
Chun-Lin Su ${ }^{1,2 *}$, Yu-Lin Tsai ${ }^{3}$, Yuan-Yang Cheng ${ }^{3,4,5}$ and Shin-Tsu Chang ${ }^{3,6,7 *}$ \\ ${ }^{1}$ Department of Education, Taichung Veterans General Hospital, Taiwan \\ ${ }^{2}$ School of Medicine, Chung Shan Medical University, Taiwan \\ ${ }^{3}$ Department of Physical Medicine and Rehabilitation, Taichung Veterans General Hospital, Taichung, Taiwan \\ ${ }^{4}$ School of Medicine, National Yang-Ming University, Taiwan \\ ${ }^{5}$ Center for Geriatrics and Gerontology, Taichung Veterans General Hospital, Taiwan \\ ${ }^{6}$ Department of Physical Medicine and Rehabilitation, Tri-Service General Hospital, Taiwan \\ ${ }^{7}$ School of Medicine, National Defense Medical Center, Taiwan \\ * Contributed equally
}

*Corresponding author: Shin Tsu Chang, Taichung Veterans General Hospital, Taiwan.
Received Date: January 08, 2021

Published Date: January 29, 2021

\begin{abstract}
Chronic knee pain is a common degenerative disorder amongst the elderly, and differential diagnosis through imaging remains crucial. Bone marrow edema represents an increase of water content in the normally fatty bone marrow. The size of the bone marrow edema (BME) is associated with the development of knee pain in Osteoarthritis $(\mathrm{OA})$. Bone marrow edema cannot be seen on plain radiography, and while a SPECT/CT scan is more sensitive than MRI in detection of bone marrow edema, the definite diagnosis of bone marrow edema is dependent upon MRI. SPECT/CT is the combination of 3D scintigraphy and computed tomography, which provides more specific anatomical localization and improved sensitivity. It is a valuable tool for the early evaluation of knee pain, as the increase of bone tracer uptake can be seen in bone marrow edema, cartilage lesions, meniscus tear or derangement and valgus/varus alignment. Hence acquiring information from both MRI and SPECT/CT can help determine the optimal treatment. We present a case of chronic knee pain as evaluated by MRI and SPECT/CT.
\end{abstract}

Keywords: Bone marrow edema; SPECT/CT; Meniscus tear; Knee; MRI; Chronic knee pain

\section{Introduction}

Chronic knee pain, defined as pain of at least three months duration, can be caused by untreated internal or external knee derangements, osteoarthritis, or inflammatory disorders, and its lifetime prevalence is $54 \%$ [1], while the prevalence of chronic pain has progressively increased in a significant manner [2]. The differential diagnosis of chronic knee pain is complicated. It is essential to acquire a patient's history, perform a physical examination and undergo an image study for making a diagnosis. We demonstrate the case of a patient experiencing with chronic knee pain for eight months caused by degenerative bone marrow edema and meniscus tear and introduce the significance of MRI and bone scintigraphy during diagnosis.

\section{Case Presentation}

We present a 64-year old female with an underlying disease of hyperlipidemia under Ezetrol 10mg QD and Crestor 10mg QD, having a personal history of degeneration of the lumbar spine. She suffered from right knee pain for 10 months, including morning pain 
and stiffness. She reported having a limit to her walking distance due to this right knee pain. She had previously been treated at Tung's Taichung Metro Harbor Hospital and other clinics, but the above symptoms did not improve even after undergoing physical therapy, prolotherapy and hyaluronic acid injection. Additionally, she reported headaches and soreness over the right subscapular area. She also experienced pain arising from the heel to the top of her head while walking, as well as intermittent low back pain.

She visited our Outpatient Department on 2020/09/23, where a physical examination showed local tenderness of the skull, R't hip/joint, lower back region and both Sacroiliac (SI) joints. Results from a bilateral Gaenslen's test (+), Patrick test (-), Fortin finger sign (-), revealed stooping posture and limited ADL function. A knee X-ray showed the presence of a marginal spur formation with focal joint space narrowing of the right knee, in favor of the onset of early osteoarthritis. (Figure 1) Based upon the patient's multiple discomforts in not only the right knee, a complete body bone scan with SPECT/CT was performed on 2020/10/02 (Figure 2), where a significantly increased bone turnover at the medial condyle of the right tibia was found.

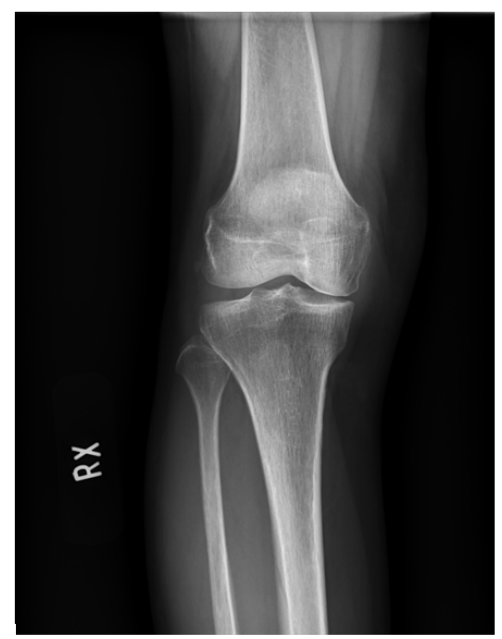

Figure 1: Knee plain radiography with the presence of marginal spur formation with focal joint space narrowing of the medial side of the right, in favor of early osteoarthritis.
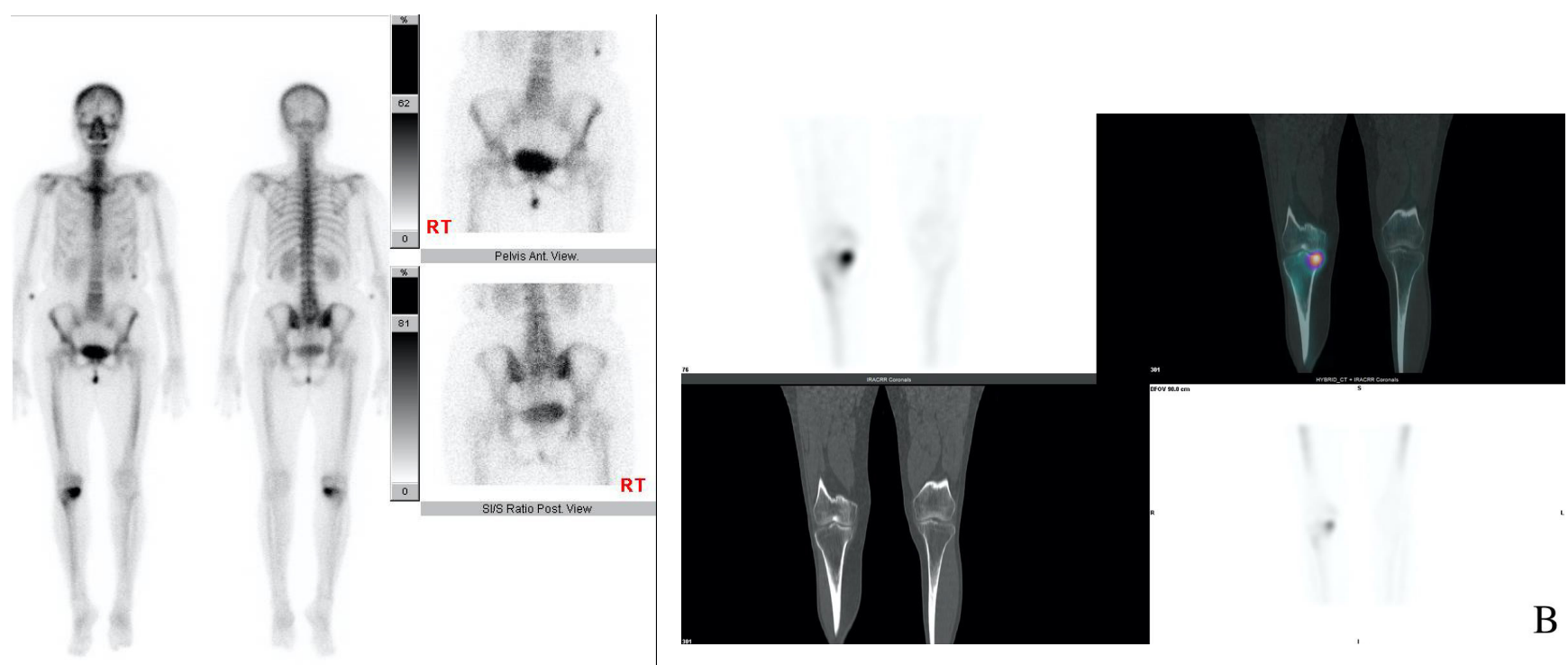

A

Figure 2: Whole body skeletal scintigraphy.

A: Whole body skeletal scintigraphy. Significantly increased bone turnover at the medial condyle of the right tibia, the anterior aspect of the left 10th rib and bilateral SI joints.

B: SPECT/CT image with significantly increased bone turnover at the medial condyle of the right tibia. 

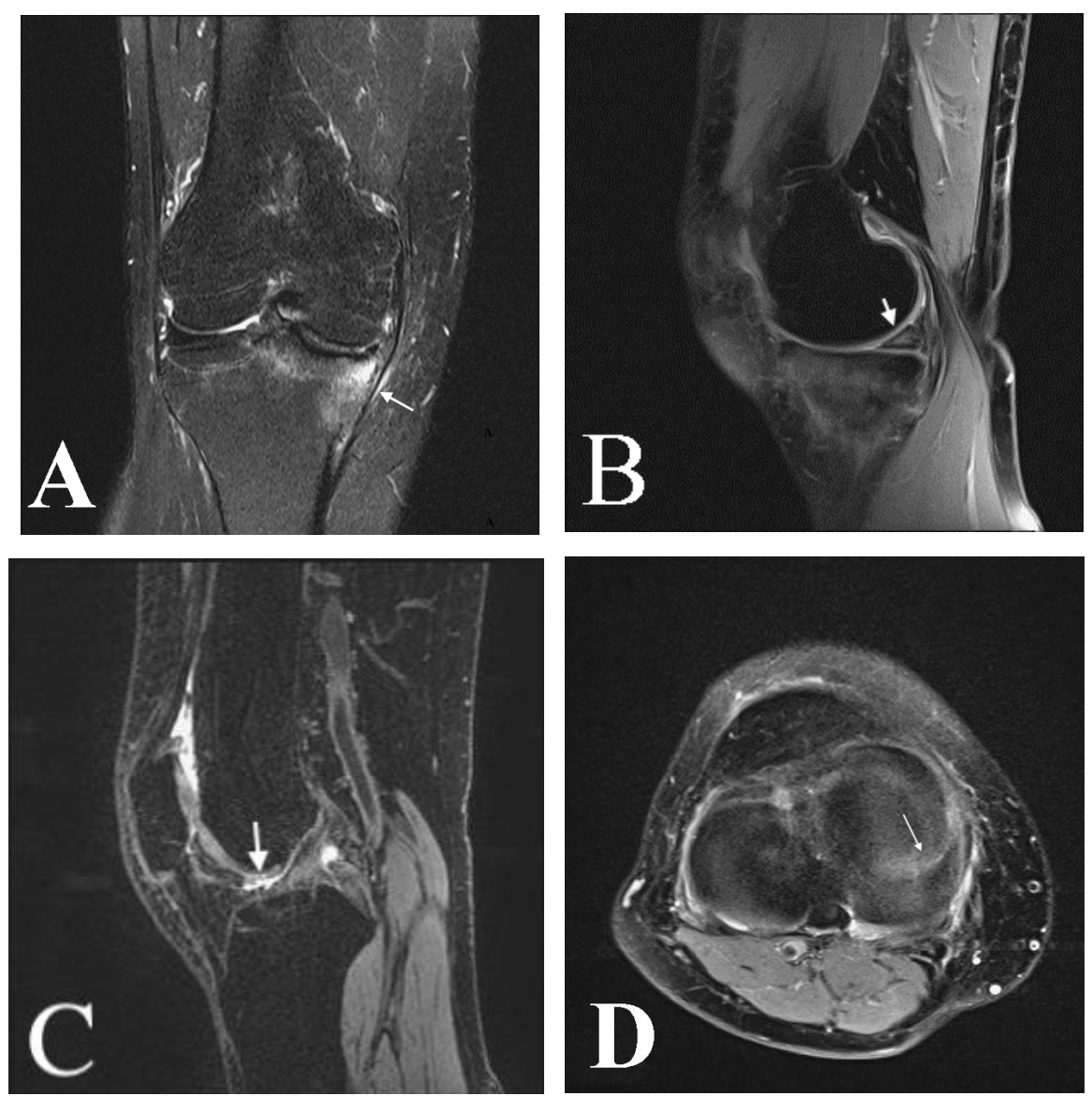

Figure 3: MRI of the right knee of the case.

A: MRI T2WI coronal image shows Grade 4 chondromalacia change with irregular subchondral plate and bone marrow edema (arrow) of the medial tibial condyle.

B: MRI PDWI fat-suppressed parasagittal image shows degenerative horizontal tear (arrow) of the posterior horn and wearing of the body of the medial meniscus. The lateral meniscus is unremarkable.

C: MRI T2WI parasagittal image shows intermediate signal intensity of the ACL (arrow), where a chronic partial tear is suspected. Grade 1 partial tear of the tibial end MCL.

D: MRI PDWI fat-suppressed transverse image shows a degenerative horizontal tear (arrow) of the posterior horn and wearing of the body of the medial meniscus.

Due to the above findings, she was admitted to our medical facility on 2020/10/12. A physical examination revealed local tenderness over the bilateral ischial tuberosity, right hip joint, and right medial knee, with results from a FADIR test (+) and L't knee grinding test $(+)$. Otherwise, there were no signs of body weight change, night sweating or low grade fever. Results from a FABER/Patrick test, Clarke test, McMurray test, Lachman test, and Anterior draw test were all negative. The patient's blood tests were unremarkable.

We arranged a sonography for her buttock ache, which revealed bursitis over the bilateral ischial tuberosity, and prolotherapy was performed without complications.

MRI of the knee was arranged on 2020/10/15 and revealed right knee synovitis, partial tear of the ACL and a grade 1 partial tear of the tibial end MCL, a degenerative horizontal tear of the posterior horn and wearing of the body of the medial meniscus, as well as grade 4 chondromalacia change with an irregular subchondral plate and bone marrow edema of the medial tibial condyle. (Figure 3) We consulted orthopedic physicians who arranged for arthroscopic meniscal repair with a right high tibial osteotomy and arthroscopic medial meniscus repair of right knee on 10/19. The findings showed right OA knee with medial compartment involvement and degenerative horizontal tear of the posterior horn and wearing of body of the medial meniscus, and the process of surgery was performed smoothly without any complications. She was then discharged on 2020/11/06 experiencing only minimal pain.

\section{Discussion}

For this patient, we performed a bone scan for initial screening regarding knee pain, where increased bone turnover at the right medial knee was found. A knee MRI then led us to the diagnosis of a meniscus tear, BME, an ACL and MCL tear. Based upon the image findings from the bone scan and MRI, we were able to conclude that there was a significant correlation between bone marrow edema and the meniscus tear. 
BME represents an increase of water content in the normally fatty bone marrow. It is commonly found in osteoarthritis, rheumatoid arthritis, osteoporosis, trauma, infections, ischemia, and neoplasm Manara M and Varenna M [3], with the most common cause being trauma [4]. The available evidence shows that trauma would contribute to capillary leakage, which is the primary cause of fluid accumulation in the extracellular marrow space and can be induced by an increased delivery and decreased clearance of the flow of blood [5]. The size of the BME is associated with the extent of knee pain in OA [6]. The radiographic features of BME in MRI are intermediate or low signal intensity on $\mathrm{T} 1$ weighted images and a high signal intensity on fat-suppressed, T2 weighted and Short Inversion Time Inversion Recovery (STIR) compared with those of normal bone marrow [3]. However, it cannot be seen on plain radiography Starr AM, et al. [7], and while SPECT/CT is more sensitive than MRI in the detection of BME, a definite diagnosis of bone marrow edema relies on MRI [8]. The condition of the patient in our case is compatible with the case of Hirschmann A and Hirschmann MT [8].

Lateral and medial menisci are critical components of a healthy knee joint. Both menisci are crescent-shaped, highly hydrated fibrocartilage situated between their corresponding femoral condyle and tibial plateau. Acute tears occur most often due to twisting injuries, while chronic degenerative tears occur in older patients and can occur with minimal twisting or stress. MRI parameters, PDWI, T1WI, and gradient echo, are the preferred imaging modalities for evaluating meniscus tears, which are confirmed by intrameniscal changes Englund M, et al. [9] and graded according to the Whole-Organ MRI Score (WORMS) method $[10,11]$. We did not use this method in our case. Instead, increased bone tracer uptake could be seen on the meniscus tear from SPECT/ CT images. Englund M, et al. [11] showed that medial meniscus injury is very common amongst those with symptomatic $\mathrm{OA}$, and meniscus derangement is upstream in a chain of events that leads to the development of bone marrow lesions. The team also claimed that there was no significant correlation between meniscal damage and the extent of knee pain. Therefore, asymptomatic patients with MRI meniscus derangements can be targeted for prevention of bone marrow lesions [11].

Skeletal scintigraphy has been increasingly recognized in clinical practice, particularly amongst orthopedic surgeons [8]. Intravenous administration of $99 \mathrm{mTc}$ MDP or HMDP is rapidly absorbed by bone tissue, then 3-phase (blood flow, blood pool/ soft tissue phase and bone phase) dynamic imaging is performed by recording radiotracer emissions through a gamma camera, with 2-dimensional (planar image) or 3-dimensional (SPECT) data being generated [12]. Increased bone tracer uptake implies hyper-perfusion, an increase in vivo loading, and bone remodeling. SPECT/CT is the combination of 3D scintigraphy and Computed Tomography, with CT images shown as grayscale background and
SPECT/CT as color graded overlay, while anatomical localization can be more specific, and the diagnostic sensitivity can improve significantly [13-15]. SPECT/CT is a valuable tool for early evaluation of knee pain, the increase of bone tracer uptake can be seen in BME, cartilage lesions, meniscus tear or derangement and valgus/varus alignment [8]. Moreover, SPECT/CT can also be useful for the evaluation of post-knee surgery recovery.

Depending on the tear, its location, and size of the tear, the main choice of treatment for degenerative meniscus tears are arthroscopic meniscectomy and conservative treatment without meniscal surgery [16]. However, several studies have shown that arthroscopic meniscectomy is not superior to conservative treatment. According to the 2016 ESSKA Meniscus Consensus Project Beaufils P, et al. [17], a plain radiograph should be the first line of assessment, with MRI being the complementary examination, while the first line of treatment is physiotherapy and the possible addition of intra-articular injections for a period of at least three months. If the treatment fails then MRI is indicated to evaluate the evidence of advanced $\mathrm{OA}$, and if no evidence of $\mathrm{OA}$ is found, arthroscopic partial meniscectomy should be proposed. If evidence of $\mathrm{OA}$ is discovered, treatment for $\mathrm{OA}$ should be considered firstline. If there are signs of mechanical symptoms (such as lack of range of motion, daily joint catching, and joint locking for more than 2 seconds over a period of at least a week), surgery can be proposed earlier. However, a clear definition of "mechanical symptoms" is yet to be defined. In our case, the patient had limited walking distance ability due to knee pain, hence surgery intervention was performed earlier, with the patient experiencing greater benefits from the surgery.

\section{Conclusion}

Traditionally, a plain radiograph and MRI is indicated for the evaluation of chronic knee pain, while we can conclude that SPECT/ CT provides valuable information for the screening of bone marrow edema and meniscus tear. In certain cases, a radiograph combined with SPECT/CT and MRI can help generate the optimal treatment, as we discovered in our case.

\section{Acknowledgement}

None.

\section{Conflict of Interest}

No conflict of interest.

\section{References}

1. Baker P, Reading I, Cooper C, Coggon D (2003) Knee disorders in the general population and their relation to occupation. Occup Environ Med 60(10): 794-797

2. Nguyen UDT, Zhang Y, Zhu Y, Niu J, Zhang B, et al. (2011) Increasing prevalence of knee pain and symptomatic knee osteoarthritis: survey and cohort data. Ann Intern Med 155(11): 725-732.

3. Manara M, Varenna M (2014) A clinical overview of bone marrow edema. Reumatismo 66(2): 184-196. 
4. Fowkes LA, Toms AP (2010) Bone marrow oedema of the knee. Knee 17(1): 1-6.

5. Eustace S, Keogh C, Blake M, Ward RJ, Oder PD, et al. (2001) MR Imaging of Bone Oedema: Mechanisms and Interpretation. Clin Radiol 56(1): 4-12.

6. Felson DT, Chaisson CE, Hill CL, Totterman SM, Gale ME, et al. (2001) The Association of Bone Marrow Lesions with Pain in Knee Osteoarthritis. Ann Intern Med 134(7): 541-549.

7. Starr AM, Wessely MA, Albastaki U, Pierre-Jerome C, Kettner NW. (2008) Bone marrow edema: Pathophysiology, differential diagnosis, and imaging. Acta radiol 49(7): 771-786.

8. Hirschmann A, Hirschmann MT (2016) Chronic knee pain: Clinical value of MRI versus SPECT/CT. Semin Musculoskelet Radiol 20(1): 3-11.

9. Englund M, Guermazi A, Lohmander SL (2009) The role of the meniscus in knee osteoarthritis: A cause or consequence? Radiol Clin North Am 47(4): 703-712.

10. Peterfy CG, Guermazi A, Zaim S, Tirman PF, Miaux Y, et al. (2004) WholeOrgan Magnetic Resonance Imaging Score (WORMS) of the knee in osteoarthritis. Osteoarthritis Cartilage 12(3): 177-190.

11. Englund M, Niu J, Guermazi A, Roemer FW, Hunter DJ, et al. (2007) Effect of meniscal damage on the development of frequent knee pain, aching, or stiffness. Arthritis \& Rheum 56(12): 4048-4054.
12. Montilla Soler J, Makanji R (2017) Skeletal Scintigraphy. Cancer Control 24(2): 137-146.

13. Mucha A, Dordevic M, Hirschmann A, Rasch H, Amsler F, et al. (2015) Effect of high tibial osteotomy on joint loading in symptomatic patients with varus aligned knees: A study using SPECT/CT. Knee Surg Sports Traumatol Arthrosc 23(8): 2315-2323.

14. Chang ST, Liu CC, Yang WH (2019) Single-photon emission computed tomography/computed tomography (hybrid imaging) in the diagnosis of unilateral facet joint arthritis after internal fixation for atlas fracture. HSOA J Med Study Research 2: 010.

15. Chang ST, Tsai WY (2020) Abrupt swollen bump of the shoulder girdle joint as the first presentation of flare-up in a case of axial spondylarthritis. Canadian J Biomedical Research Technology 3(5): 1-5.

16. Fox MG (2007) MR Imaging of the meniscus: Review, current trends, and clinical implications. Magn Reson Imaging Clin N Am 15(1): 103-123.

17. Beaufils P, Becker R, Kopf S, Englund M, Verdonk R, et al. (2017) Surgical management of degenerative meniscus lesions: The 2016 ESSKA Meniscus Consensus. Joints 25(2): 335-346. 